\title{
Treatment of epilepsy: whose right is it anyway?
}

\author{
N BUCHANAN
}

While the management of patients with epilepsy has considerably improved over the past two decades, several problems remain. The thoughts presented here are the result of several years' experience in dealing with patients, both adults and children, either to rationalise their drug treatment or because they have been dissatisfied for one reason or another with the management of their epilepsy. These problems include:

(1) Polypharmacy.

(2) The possible overuse of therapeutic drug monitoring.

(3) Drug non-compliance.

(4) Should all seizures be treated or eradicated ?

(5) Educational aspects both with regard to the patient and the doctor.

(6) The patient's understanding of epilepsy.

\section{Polypharmacy}

While there are more reports on the advantages of anticonvulsant monotherapy, ${ }^{1}$ there is little doubt that this concept has not been espoused by all prescribers. Reports, and practical experience, would suggest that roughly $60-80 \%$ of patients with epilepsy can be well controlled with one appropriately chosen drug. By the same token, there is no doubt that certain patients will need more than one drug, but rarely more than three. Our own group has recently been concerned in what was initially an academic exercise-namely, trying to reduce the number of anticonvulsant drugs being administered to epileptic patients in institutions. In this "hard core" group of 20 epileptic patients who were initially taking an average of four anticonvulsants each, treatment has been reduced dramatically over 12 months. Three patients have ceased treatment entirely and have had no seizures.

\footnotetext{
Paediatric Pharmacology Unit, Department of Paediatrics, Westmead Centre, Westmead, NSW 2145, Australia

N BUCHANAN, PHD, FRACP, director of paediatrics
}

One patient having had her treatment rationalised is now taking only two drugs, has left the institution, and is working as a librarian. Of the remaining 16,15 have had their drugs reduced to two compounds, all administered on a twice daily basis, but in one patient it was not possible to effect any change. In none of these patients has seizure control deteriorated, in most it has improved, but more importantly, the quality of life in terms of awareness and alertness has been enhanced. If this can be achieved in such a group of patients most less severe epileptics may be controlled with one judiciously chosen drug.

\section{Possible overuse of therapeutic drug monitoring}

Regrettably, as has occurred so often with the advent of technological advances, the measurement of serum drug concentrations has become as commonplace as the assessment of "routine biochemistry." It is not uncommon for patients to question the validity of such assays on the basis that they are well controlled (seizure free) and therefore wonder why blood tests are necessary. The easy availability of serum anticonvulsant assays that allows all laboratories, however small, to carry out these tests, has made this possible. Well-defined indications exist for measuring serum anticonvulsant concentration ${ }^{2}$; the fact that a patient is receiving a drug is in itself no indication for such a measurement, nor do patients who are well controlled require such measurements. "Routine drug level checks" are unacceptable.

\section{Drug non-compliance}

About $40 \%$ of epileptics are non-compliant. ${ }^{3}{ }^{4}$ The traditional medical interpretation of this phenomenon is that these patients are "naughty" and "irreverently" ignoring medical advice. An alternative interpretation is that they are in fact telling us, medical practitioners, either that they do not agree with the proposed treatment or that they deem it inappropriate. The 
reasons for this are either that the side effects of the drugs being prescribed are in their opinion worse than the disorder for which they are being prescribed or that their seizure frequency is in fact such that they would rather not take drugs and have the occasional fit. Non-compliance is a phenomenon that needs to be respected by the medical practitioner and the message that the patient is giving the doctor by this practice needs to be correctly interpreted.

\section{Should all seizures be treated or eradicated?}

Epilepsy is a disorder that, par excellence, requires individualised treatment. Firstly, it is inappropriate to talk of an "epileptic patient." There are numerous forms of epilepsy, all of which differ in aetiology, frequency of seizures, disruption of lifestyle, and prognosis. The time has come to no longer speak of epilepsy in a general way but to be more specific, and to state that patients have, for example, temporal lobe epilepsy, tonic/ clonic seizures, or absence seizures. While it has traditionally been taught, and it is tempting to try, to eradicate all seizures in each epileptic patient, the reality is that in some patients this may be extremely difficult and that the price paid for eradicating seizures may be greater in terms of adverse drug effects than it is worth. It is extremely important that a consensus be reached between the doctor and each individual patient as to the degree of seizure control required by that individual. When such a proposal is put to patients, depending on their degree of selfconfidence, the nature of their seizures, and the drugs that they are taking, their response may often be extremely positive. For example, many patients with petit mal epilepsy or with nocturnal seizures, may be interested in reaching a compromise, where they suffer no drug side effects but have the occasional seizure. It is the patient's right to decide on the degree of seizure control that he desires and not a prerogative of the doctor to insist on complete eradication of seizures.

\section{Education}

Many epileptic patients feel isolated in society and discriminated against by virtue of their epilepsy. There is little doubt that the lay public see epilepsy as a frightening disorder and tend to shun the company of those afflicted. While it would be nice to believe that education of the community as a whole would remove these prejudices, it has to be accepted that this is a longterm aim. In the interim, and possibly of equal importance, is the need to enhance self-esteem for the epileptic person and to increase the education of doctors.

One of the most important factors in the educational process is to get the epileptic patient, as much as possible, to take responsibility for managing his disorder. Doctors should realise that unless they themselves have epilepsy they have no experience of having seizures and their social consequences. It is therefore mandatory for them to accept that the patient under their care is the sole arbitrator of this particular prerogative. The acceptance of this principle implies that the patient or parent, if of sound mind, has prime responsibility for managing the disorder. I have found that roughly $75^{\circ}$ of patients/parents, when asked if they would like to control the drug treatment for themselves or their child, willingly accept this responsibility. Indeed, it is a matter of surprise and sometimes irritation to them that this role has previously been expropriated by their doctor. The prime mover in controlling treatment, its dosage and frequency, should ideally be the patient, for it is he who is aware of his seizures, their frequency, and their implications for his lifestyle. There are, however, some patients who are unwilling to accept this responsibility and would prefer to adopt a traditional medical model whereby the physician gives them instructions that they "faithfully" carry out. It is presumptious of the medical profession to take over the treatment of patients who are more aware of the social implications of their disorder than are their doctors.

If it is accepted that in certain, or possibly many, instances the epileptic person is better informed about the consequences of his epilepsy than are we, the doctors, then it is important to accept that education of the doctor by the patient is also required. This may best be achieved by listening to patients who often have illuminating views about their epilepsy, its significance, and its management.

\section{Understanding of epilepsy}

One of the most important factors in eradicating prejudices concerning epilepsy is to inform the epileptic patient of the nature, prognosis, and therapeutic requirements for their particular form of epilepsy. To achieve this, patients require both detailed and recurrent information. To many doctors, this is irksome, both in time and practicality. Epileptic patients on certain occasions may be demanding, either in terms of seizure control or by virtue of their social problems. It is important for the doctor to accept that he, as an individual, may have limitations in dealing with these particular problems. Should this be the case, it is imperative to suggest to the patient that it might be more appropriate for him to seek advice from an individual who has a particular interest in managing epilepsy, be it either in terms of anticonvulsant medication or the social aspect thereof.

\section{Conclusion}

Many problems related to managing the epilepsies have been discussed. Epilepsy is a personal disorder and a model of patient self-management, which "is his/her right anyway."

\section{References}

${ }^{1}$ Reynolds EH, Shorvon SD. Monotherapy or polytherapy ? Epilepsia 1981; 22:1-10.

2 Koch-Weser J. Serum drug concentrations in clinical practice. Therapeutic Drug Monitoring 1981 ;3:3-16.

${ }^{3}$ Freiman J, Buchanan N. Drug compliance and therapeutic considerations in 75 black epileptic children. Cent Afr $\mathcal{F}$ Med 1978;24:136-40.

+ Buchanan N, Shuenyane E, Mashigo S, Mtangai P, Unterhaltar B. Factors affecting drug compliance in ambulatory black urban patients. $S$ Afr Med F 1979;55:368-73.

(Accepted 6 November 1981)

In what circumstances should potassium supplements be used for patients receiving diuretic drugs?

Both the thiazide and loop diuretics increase urinary potassium loss and may cause depletion of total body potassium. This is particularly likely if a brisk diuresis occurs or if the oedema is accompanied by secondary hyperaldosteronism. Potassium depletion may also occur when diuretic treatment is continued after clearance of the oedema. In these circumstances the diuretic may cause sodium depletion, which in turn produces secondary hyperaldosteronism and accentuation of the urinary loss of potassium. In all of these instances potassium supplementation is essential, and in patients in whom oedema and secondary hyperaldosteronism coexist it is preferable to start treatment with potassium sparing diuretics. When moderately active thiazide diuretics are used for the long-term treatment of hypertension, it is not usually necessary to prescribe potassium supplements provided the patient takes an adequate diet that contains about $100 \mathrm{mmol} / \mathrm{l}$ of potassium a day. It should be borne in mind that the diet of elderly patients may lack in this respect, in which case either the diet needs to be improved or potassium supplements may be given if long-term diuretic treatment is really necessary.-A W ASSCHER, professor of renal medicine, Cardiff. 